$\Rightarrow$ MICROBIOME

\section{Anelloviridae go viral}

The bacterial component of the human microbiome has been extensively studied, whereas little is known about the viral component and the factors that shape it. A study

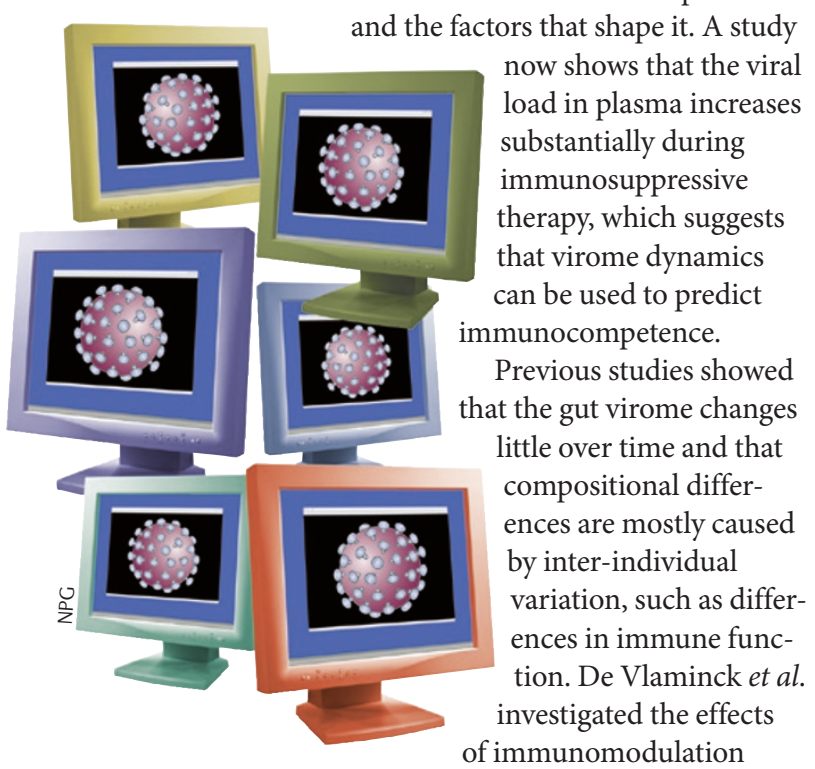

by analysing the plasma virome of 96 organ transplant patients who received immunosuppressive drugs to prevent organ rejection. Shotgun sequencing of cell-free DNA from plasma samples that were collected over several months post-transplantation showed that viral loads increased, on average, by a factor of 7.4 (which correlates with a parallel increase in immunosuppression), whereas the bacterial composition remained unchanged.

The increased viral load could be partly explained by the expansion of members of the Anelloviridae - which is a recently discovered virus family that is often present at low levels in healthy individuals. In some patients, more than $90 \%$ of all viral sequences originated from Anelloviridae, particularly in cytomegalovirus (CMV)-positive patients who were treated with valganciclovir or acyclovir to prevent CMV reactivation. These drugs did not suppress the expansion of the virome but inhibited the replication of susceptible viruses (including $\mathrm{CMV}$ ), whereas anelloviruses were unaffected. In patients who did not receive antiviral drugs, anelloviruses also dominated the virome; however, other virus families also expanded. Several months posttransplantation, when the immunosuppressive treatment was slowly reduced, anellovirus loads started to decline, which indicates that control of anellovirus replication is a measure of immunocompetence. Indeed, in patients who showed signs of transplant rejection owing to insufficient immunosuppression, anellovirus loads were significantly lower than in non-rejecting patients.

Taken together, these results show that the immune system has an important role in determining the size and composition of the virome, and that changes in viral load - particularly anellovirus load could potentially be used to measure immunocompetence, which might reduce the need for diagnostic organ biopsies in transplant patients.

\section{Ursula Hofer}

ORIGINAL RESEARCH PAPER De Vlaminck, I. et al. Temporal response of the human virome to immunosuppression and antiviral therapy. Cell 155, 1178-1187 (2013) 